\title{
Short Term Effects of Rilmenidine on Left Ventricular Hypertrophy and Systolic and Diastolic Function in Patients with Essential Hypertension
}

\section{Comparison with an Angiotensin Converting Enzyme Inhibitor and a Calcium Antagonist}

\author{
Lale KolDAs, ${ }^{1} \mathrm{MD}$, Faruk AYAN, ${ }^{1} \mathrm{MD}$, and Baris IKITIMUR, ${ }^{1} \mathrm{MD}$
}

\begin{abstract}
SUMMARY
The short term (three months) effects of rilmenidine on systemic hypertension induced left ventricular hypertrophy $(\mathrm{LVH})$ and left ventricular systolic and diastolic functions in comparison with those of perindopril and nifedipine-slow release (SR) formulation were studied. The short term effects of rilmenidine on biochemical parameters and lipid profiles were evaluated.

Sixty patients (39 men, 21 women) with a mean age of $59 \pm 14$ years and with mild to moderate systemic arterial hypertension were enrolled in three groups. The first group received $1 \mathrm{mg} /$ day of rilmenidine, the second group $4 \mathrm{mg} /$ day of perindopril, and the third group $20 \mathrm{mg} / \mathrm{day}$ of nifedipine SR. All drugs induced a similar decrease in systolic and diastolic blood pressure (BP) values. Left ventricular mass (LVM) and LVM index decreased equally in all groups associated with a significant increase in the E/A ratio. The ratio between the reduction in LVM and decrease in mean arterial pressure $(\mathrm{LVM} / \mathrm{mmHg}$ ) was higher in groups 1 and 2. Negative correlations between LVM and LVMI, E/A, and the $d v / d t$ ratio were obtained. Rilmenidine did not change the blood chemistry and lipid profile values.

Despite its neutral effect on lipid profile and biochemical parameters, rilmenidine is as effective as perindopril and nifedipine in controlling hypertension and decreasing left ventricular hypertrophy. (Jpn Heart J 2003; 44: 693-704)
\end{abstract}

Key words: Rilmenidine, Left ventricular hypertrophy, Echocardiography, Lipid profile, Blood chemistry

MANY antihypertensive drugs have proven effective in reversing left ventricular hypertrophy $(\mathrm{LVH})$, which is an independent risk factor for cardiovascular morbidity and mortality. ${ }^{1}$ Both development and regression of LVH influence left ventricular function and alter diastolic performance and coronary flow reserve. Dahlöf, et al have demonstrated that a comparable fall in blood pressure induced

\footnotetext{
From ${ }^{1}$ the Department of Cardiology, Istanbul University Cerrahpasa Medical Faculty, Istanbul, Turkey. Address for correspondence: Lale Koldas, MD Valikonagi Cad. No:127 D:16 Nisantasi, Istanbul, Turkey. Received for publication November 15, 2002.

Revised and accepted January 16, 2003. 
by different antihypertensive drugs has different effects on LVH. This indicates that the ability of each antihypertensive drug to reverse LVH must be demonstrated and cannot be extrapolated from the drug's antihypertensive effect. ${ }^{2)}$ It is well recognized that in addition to hypertension, there are many other factors like sympathetic tonus, angiotensin II, age, blood viscosity, hormonal factors such as insulin, growth factor, thyroxin and sex hormones, contributing to the development of LVH.

The discovery of imidazoline receptors has led to the development of new antihypertensive agents like rilmenidine, a new oxazoline, acting selectively through imidazoline preferring receptors with sympatholytic antihypertensive properties through a selective action on bulbar vasomotor centers and on peripheral receptors. ${ }^{3)}$ In several studies rilmenidine has been shown to reduce left ventricular mass in patients with left ventricular hypertrophy when given on a regular basis for a one year period. ${ }^{4,5)}$ The efficacy of rilmenidine in lowering blood pressure when given for a four week time period had been demonstrated previously in a study by Ostermann, et al. ${ }^{6}$ It has been observed that regression of left ventricular hypertrophy takes a long time and depends not only on the drug regimen chosen but also on the duration of treatment. ${ }^{7)}$ The salutary effects of rilmenidine on left ventricular hypertrophy observed over a time period as long as one year raises the question whether this regression effect can be achieved during a shorter time period in which rilmenidine's antihypertensive effect has already been proven. In clinical practice, a serious problem of cost-effectiveness exists in the area of initial evaluation and re-assessment of LVH in patients with systemic arterial hypertension. Would it be justifiable to expect reversal of LVH in a less than one year time period of therapy? Should a physician consider changing his/her choice of antihypertensive therapy if the expected reversal of LVH is not observed despite achievement of acceptable blood pressure levels? Although this study was not designed to, nor does it have enough power to answer these questions, the detection of early trends in LVH reversal in hypertensive patients is most likely a good place to start.

Since in previous studies ACE inhibitors, together with diuretics, have been shown to decrease LVH far better than beta blockers and better than calcium antagonists, we compared the effects of rilmenidine to one drug from the superior class (ACE inhibitor), and to one from the moderate performer group (calcium antagonist). ${ }^{8)}$

This study was designed to assess the short term (three months) effects of rilmenidine on systemic hypertension-induced $\mathrm{LVH}$ and left ventricular systolic and diastolic functions; to compare these effects with those of the angiotensin converting enzyme inhibitor (ACE-I) perindopril and the calcium antagonist (CAA) nifedipine -slow release formulation, both of which are known to reverse 
$\mathrm{LVH}$; and to evaluate the efficacy, tolerability, and short term effects of rilmenidine on biochemical parameters and the lipid profile.

\section{METHODS}

A total of sixty patients (39 men, 21 women) with a mean age of $59 \pm 14$ years were included in the study. All of the patients had mild to moderate hypertension with initial blood pressure systolic values $<190 \mathrm{mmHg}$ and diastolic values between 90-110 mmHg and accompanying left ventricular hypertrophy. Any obvious etiologies of left ventricular hypertrophy other than arterial hypertension such as significant valvular heart disease on echocardiography or hypertrophic cardiomyopathy, as well as secondary hypertension, prior myocardial infarction, a history or clinical evidence of coronary artery disease, diabetes mellitus, renal insufficiency, or regularly performing exercise were reasons for exclusion from the study. Blood pressure was between $150 \mathrm{mmHg}$ and $190 \mathrm{mmHg}$ systolic, and between $90 \mathrm{mmHg}$ and $110 \mathrm{mmHg}$ diastolic on at least 5 consecutive measurements. Readings were obtained after 10 minutes in the supine position and after 2 minutes standing, by means of a standard sphygmomanometer with a cuff of appropriate size, following the recommendations of the American Heart Association. ${ }^{9)}$ The initial values of left ventricular mass (LVM) were > 259 grams in men and $>166$ grams in women with concurrent left ventricular mass index (LVMI) values of $>130 \mathrm{~g} / \mathrm{m}^{2}$ for men and $>110 \mathrm{~g} / \mathrm{m}^{2}$ for women. Each patient satisfied the echocardiographic criteria of LVH. ${ }^{10}$

Study protocol: After a complete physical examination, blood pressure measurements, electrocardiogram (ECG) recordings and blood chemistry analyses were performed and the patients underwent a ten day wash-out period, free of any antihypertensive medications. On the day of randomization all patients underwent baseline measurements including blood pressure determination, ECG recording, blood chemistry analysis, and transthoracic echocardiography. M-mode and pulsed Doppler studies performed together with a two-dimensional (2-D) echocardiographic study for measurement of left ventricular (LV) wall thickness and dimensions in order to calculate LV mass (LVM) and mass index (LVMI) for the evaluation of $\mathrm{LVH}$, left ventricular ejection fraction (LVEF) as a marker of systolic function, early (E) and late (A) peak diastolic filling velocities, E/A ratio, deceleration time $(\mathrm{dt})$ and deceleration velocity/dt ratio $(\mathrm{dv} / \mathrm{dt})$ were done to assess LV diastolic function for each patient. A Hewlett-Packard Sonos 2500 echocardiograph equipped with a 2.5/3.0 MHz transducer was used.

The subjects were randomized into three groups. The first group $(n=20$, mean age $57 \pm 14$ years) received $1 \mathrm{mg} /$ day of Rilmenidine, the second group ( $n$ $=20$, mean age $60 \pm 15$ years) received $4 \mathrm{mg} /$ day of perindopril, and the third 
group of patients ( $n=20$, mean age $59 \pm 13$ years) was given $20 \mathrm{mg} /$ day of nifedipine SR. Since most of the patients fell into the mild to moderate hypertension categories, the above-mentioned dosages which are commonly applied as initial monotherapy for this indication were selected. Blood pressure, heart rate measurements, and a tolerability questionnaire were performed in weeks 2, 4, 6, 8 and in the 12th week, when an ECG recording, blood chemistry analysis, and an echocardiogram were also repeated (Figure 1). Each drug was given as once daily tablets administered at 9 AM. None of the patients received any medication other than the study drugs throughout the study.

The effect of each drug on several biochemical parameters including urea, fasting blood glucose, sodium, potassium, chloride, ALT, AST, and lipid profile (total cholesterol, triglyceride, VLDL, LDL, HDL) were evaluated and serum values before initiation of therapy and at the end of the three month study period were compared.

Statistical analysis was performed using Student's $t$-test while comparing echocardiographic and biochemical parameters before and after drug therapy. The differences were considered to be statistically significant when a $P$ value $<$ 0.05 was obtained. The Pearson test was used to identify whether any correlation existed between LVM and the LVMI-E/A ratio, LVM and LVMI-dv/dt, and LVM and LVMI-systolic and diastolic blood pressure values.

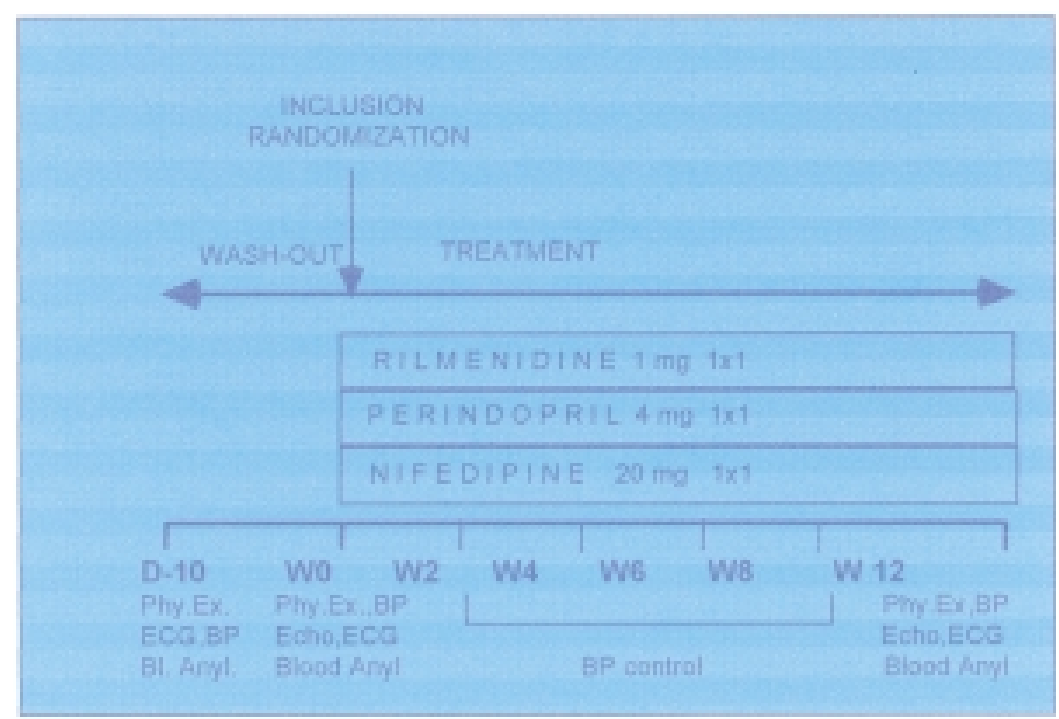

Figure 1. Study protocol 


\section{RESULTS}

All three drugs induced similar decreases in systolic and diastolic blood pressure (BP) values in standing and supine positions, first noted on the second week visit and continuing thereafter (Table I). A fall in BP to normal limits (< $140 / 90 \mathrm{mmHg}$ ) was obtained in $76 \%, 78 \%$, and $75 \%$ of patients after rilmenidine, perindopril and nifedipine SR therapies, respectively (Figures 2 and 3).

Table I. Comparison of Antihypertensive Efficacy

\begin{tabular}{lccccccc}
\hline & SBP-b & SBP-a & $\%$ & DBP-b & DBP-a & $\%$ & $* P$ \\
\hline I-Rilmenidine & $173 \pm 18$ & $141 \pm 14 *$ & 19 & $99 \pm 11$ & $84 \pm 4 *$ & 15 & $<0.05$ \\
II-Perindopril & $180 \pm 25$ & $145 \pm 15 *$ & 20 & $95 \pm 15$ & $85 \pm 7 *$ & 11 & $<0.05$ \\
III-Nifedipine & $175 \pm 27$ & $145 \pm 13 *$ & 18 & $95 \pm 13$ & $85 \pm 9 *$ & 12 & $<0.05$
\end{tabular}

$\mathrm{SBP}=$ systolic blood pressure DBP $=$ diastolic blood pressure $;-\mathrm{a}=$ after; $-\mathrm{b}=$ before therapy (I-II,I-III,II-III parameters $P>0.05$ ).

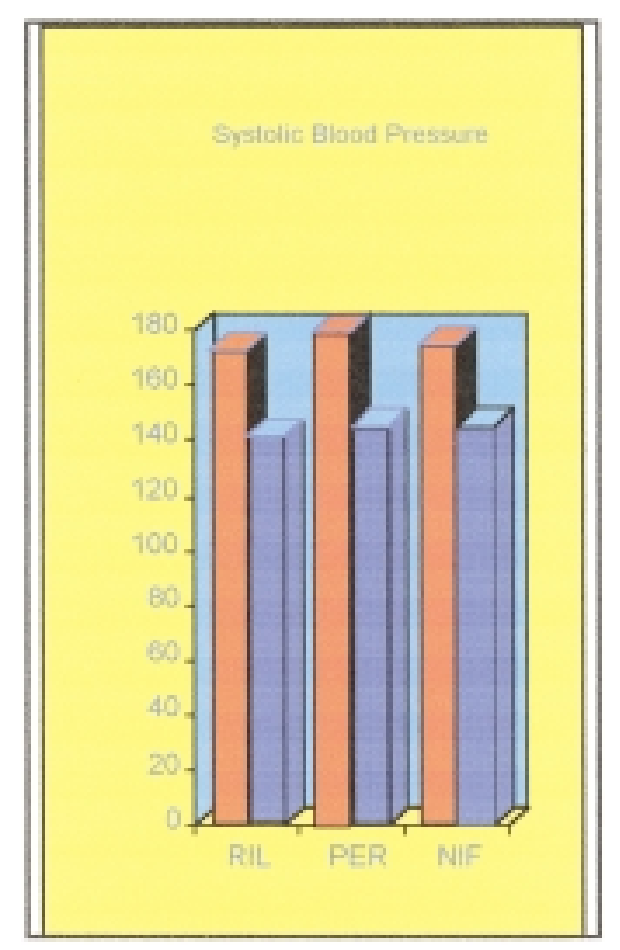

Figure 2. Comparison of the fall in ststolic blood pressure (Ril $=$ Rilmenidine group Per $=$ Perindopril group Nif $=$ Nifedipine group) Red: before, blue: after treatment. 


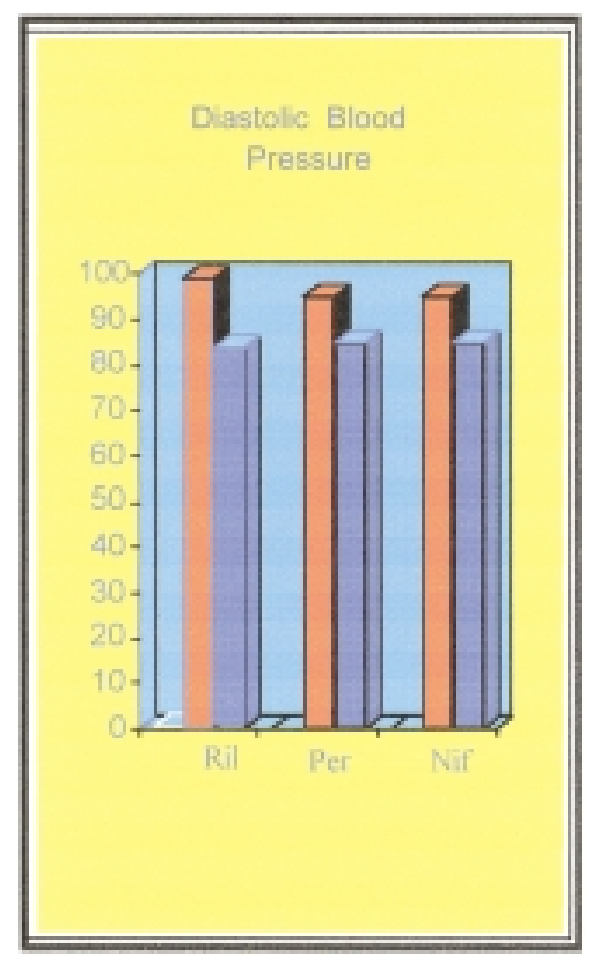

Figures 3. Comparison of the fall in diastolic blood pressure $($ Ril $=$ Rilmendine group; Per $=$ Perindopril group; Nif = Nifedipine group) Red: before, blue: after treatment.

LVM decreased significantly and equally in all 3 groups, but LVMI decreased significantly only in the perindopril and rilmenidine groups. Also, a significant increase in the E/A ratio, a well-known indicator of left ventricular diastolic function, was observed in patients receiving rilmenidine and perindopril but not in patients receiving nifedipine. The ratio between the reduction in LVM and the fall in mean arterial pressure $(\mathrm{LVM} / \mathrm{mmHg})$ was also higher in the rilmenidine and perindopril groups compared to those for the patients treated with nifedipine (Table II, Figure 4).

There were no correlations between LVM, LVMI and systolic or diastolic $\mathrm{BP}$, but negative correlations between LVM and LVMI, E/A, and the dv/dt ratio were observed. 
Table II. Comparison of Effect on Ventricular Hypertrophy and Systolodiastolic Functions

\begin{tabular}{|c|c|c|c|c|c|c|}
\hline & \multicolumn{2}{|c|}{ Rilmenidine } & \multicolumn{2}{|c|}{ Perindopril } & \multicolumn{2}{|c|}{ Nifedipine } \\
\hline & Before & After & Before & After & Before & After \\
\hline LVM (g) & $348 \pm 119$ & $331 \pm 112 *$ & $355 \pm 121$ & $339 \pm 112 *$ & $349 \pm 111$ & $338 \pm 113 *$ \\
\hline LVMI $\left(\mathrm{g} / \mathrm{m}^{2}\right)$ & $199 \pm 55$ & $189 \pm 61^{*}$ & $202 \pm 62$ & $192 \pm 58^{*}$ & $203 \pm 56$ & $198 \pm 58^{*}$ \\
\hline $\operatorname{LVEF}(\%)$ & $0.62 \pm 0.11$ & $0.59 \pm 0.10$ & $0.60 \pm 0.06$ & $0.59 \pm 0.06$ & $0.58 \pm 0.11$ & $0.62 \pm 0.05$ \\
\hline LVFS (\%) & $0.32 \pm 0.04$ & $0.34 \pm 0.06$ & $0.31 \pm 0.06$ & $0.32 \pm 0.05$ & $0.33 \pm 0.04$ & $0.35 \pm 0.06$ \\
\hline $\mathrm{E} / \mathrm{A}$ & $0.9 \pm 0.4$ & $1.3 \pm 0.5^{*}$ & $1.1 \pm 0.4$ & $1.4 \pm 0.4^{*}$ & $1.2 \pm 0.4$ & $1.2 \pm 0.4^{*}$ \\
\hline $\mathrm{dt}(\mathrm{s})$ & $0.44 \pm 0.1$ & $0.42 \pm 0.1$ & $0.43 \pm 0.1$ & $0.41 \pm 0.1$ & $0.45 \pm 0.1$ & $0.44 \pm 0.1$ \\
\hline $\mathrm{dv} / \mathrm{dt}\left(\mathrm{m} / \mathrm{s}^{2}\right)$ & $2.1 \pm 0.5$ & $2.4 \pm 0.5^{*}$ & $2.0 \pm 0.5$ & $2.3 \pm 0.4^{*}$ & $2.1 \pm 0.5$ & $2.2 \pm 0.4$ \\
\hline LVM/BP $(\mathrm{g} / \mathrm{mmHg})$ & \multicolumn{2}{|c|}{1.6} & \multicolumn{2}{|c|}{1.8} & \multicolumn{2}{|c|}{1.3} \\
\hline
\end{tabular}

$\mathrm{E}=$ Early, $\mathrm{A}=$ Late oliastolic filling velocities $; \mathrm{LVM}=$ left ventricular mass; $\mathrm{dt}=$ decceleration time $; \mathrm{dvldt}=$ decceleration velocity/decceleration ejection fraction; LVFS = left ventricular fractional shortening; LVM/BP $=$ Ratio between reduction in left ventriculer mass and the fall in mean arterial pressure.

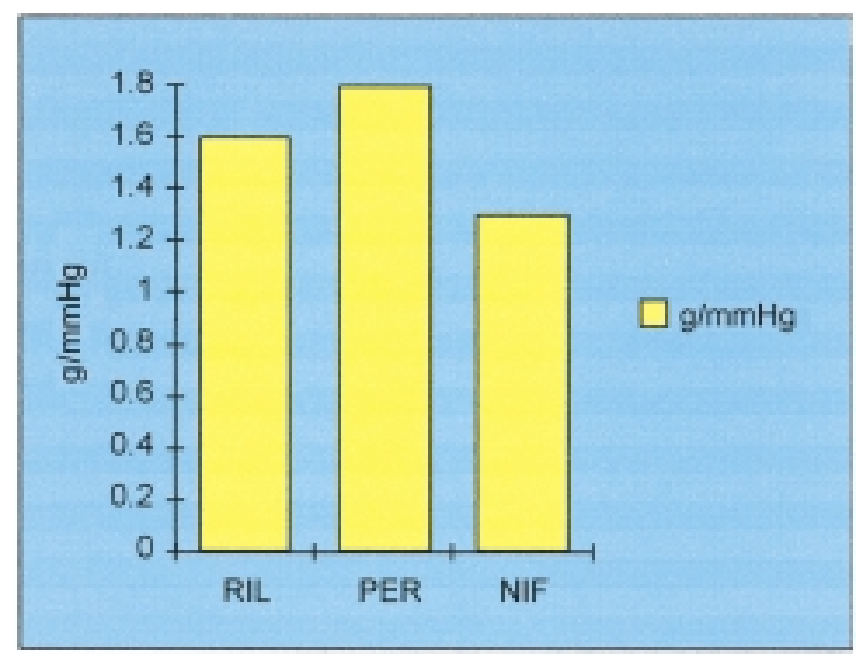

Figure 4. Ratio between the reduction in left ventricular mass (LVM) and the fall in mean arterial pressure $(\mathrm{LVM} / \mathrm{mmHg})($ Ril $=$ Rilmendine group; Per $=$ Perindopril Group; Nif = Nifedipine group).

No significant changes in heart rate (HR), cardiac output (CO) and blood glucose, urea, creatinine, SGOT, SGPT, Na, K, Cl, total cholesterol, triglyceride, HDL, LDL, or VLDL levels were obtained. A statistically insignificant increase in HDL-C level (+6\%) was observed during rilmenidine therapy (Table III). 
Table III. Effect of Rilmenidine on Biochemical Parameters and Lipid Profile

\begin{tabular}{lcc}
\hline RIL $(n=20)$ & Before & After \\
\hline Urea & $44 \pm 19.6$ & $36 \pm 12.1$ \\
Glucose & $102 \pm 76.3$ & $109 \pm 59.9$ \\
$\mathrm{Na}^{+}$ & $141.5 \pm 3.7$ & $142.7 \pm 2.7$ \\
$\mathrm{~K}^{+}$ & $4.22 \pm 0.4$ & $4.27 \pm 0.4$ \\
$\mathrm{Cl}^{-}$ & $101.3 \pm 1.2$ & $102.3 \pm 2.1$ \\
$\mathrm{ALT}$ & $19.7 \pm 8.3$ & $20.7 \pm 7.3$ \\
AST & $21.9 \pm 10.9$ & $28.4 \pm 18.8$ \\
T. Cholesterol & $228 \pm 37.7$ & $223 \pm 37.1$ \\
Triglycerides & $140 \pm 57.5$ & $141 \pm 47.2$ \\
VLDL & $31.3 \pm 13.6$ & $28.9 \pm 10.1$ \\
LDL & $149 \pm 35.2$ & $147 \pm 32.3$ \\
HDL & $42 \pm 11.4$ & $45 \pm 11.3$ \\
\hline
\end{tabular}

For all parameters compared (before therapy and after therapy), $P>0.05$

All three drug regimes were well tolerated and no important side effects, except mild ankle edema in $30 \%$ of the nifedipine patients, were noted.

\section{DISCUSSION}

Systemic arterial hypertension is a major, modifiable risk factor for cardiovascular disease. An important clinical phenomenon known to be associated with hypertension is left ventricular hypertrophy, which itself is now recognized as an independent risk factor for development of stroke, and is known to be associated with increases in total and cardiovascular mortality, sudden death, and progression to congestive heart failure. ${ }^{1,11)}$

The reason why $\mathrm{LVH}$ represents an independent cardiovascular risk factor has been attributed to several underlying pathophysiological mechanisms. First of all, an impaired systolic and diastolic function has been found to be associated with an increasing degree of LVH. ${ }^{12,13)}$ Second, reduced coronary reserve was noted in hypertensive patients even in the absence of coronary artery stenoses. ${ }^{14}$ ) Finally it has been proposed that the relation between LVH and sudden cardiac death may stem from an increase in ventricular arrhythmias. ${ }^{15)}$

Meta-analytic data have demonstrated that a comparative fall in blood pressure induced by different antihypertensives has different effects on $\mathrm{LVH}^{2}{ }^{2}$ This observation, which may be explained by the multifactorial pathogenesis of hypertension induced $\mathrm{LVH}$, further indicates that the ability of each hypertensive treat- 
ment to regress $\mathrm{LVH}$ cannot be attributed to the treatment's antihypertensive efficacy.

The autonomic nervous system plays a vital role in blood pressure control, regulating both heart rate and blood vessel diameter through, among other means, baroreceptor reflexes. Ultimately the response to raised blood pressure is a reduction in the activity of the sympathetic nervous system and a decline in blood pressure. It was also suggested that sympathetic overactivity plays a role in other pathological processes associated with elevated blood pressure including LVH, atherogenesis, ventricular arrhythmias, and metabolic disorders such as insulin resistance, hyperinsulinemia and hyperlipidemia. ${ }^{16)}$ The theory that sympathetic overactivity is a common circumstance underlying both hypertension and the above-mentioned abnormalities, which are themselves implicated in coronary heart disease, may explain why antihypertensive drugs that only act to lower blood pressure do not yield the expected reduction in coronary heart disease. In contrast, drugs that act on the sympathetic nervous system, such as centrally acting imidazoline receptors, beta-blockers, and ACE inhibitors may be expected to influence all these risk factors favorably, thereby conferring protection from the complications of hypertension beyond that achieved by mere blood pressure reduction. Imidazoline receptors, mainly the I1 sub-type, are found in the brain stem and proximal tubules in the kidneys. Renal I1 receptors are known to result in natriuresis with the assistance of the $\mathrm{Na} / \mathrm{H}$ exchanger system when activated. Sympathetic tonus decreases when I1 receptors in the brain stem are activated, resulting in peripheral vasodilatation and a decrease in peripheral vascular resistance. It is not surprising to see that rilmenidine, a structural imidazoline derivative, has more than an antihypertensive effect when the aforementioned mechanisms are considered. ${ }^{3,17}$

Rilmenidine has been shown to reduce both systolic and diastolic blood pressure in patients with essential hypertension effectively in both long term (one year) and short term (four week) therapy regimens. ${ }^{6,18)}$ An important characteristic for any antihypertensive medication is its ability to regress left ventricular hypertrophy. Rilmenidine has been proven to be effective in this task when used for a 1-year time interval. ${ }^{4,5,7)}$ The salutary effects of rilmenidine on left ventricular hypertrophy observed over a time period as long as one year raises questions whether the same effect can be observed when the drug, which is known to be effective in lowering blood pressure in the short term, is given for a 3 month period. The serious problems of timing and cost-effectiveness in the area of initial evaluation and re-assessment of patients with systemic arterial hypertension and LVH exists. If a reversal of LVH in a less than one year time period of therapy is possible and whether or not a physician considers changing his/her choice of antihypertensive therapy if the expected reversal of LVH is not observed despite 
achievement of acceptable blood pressure levels need to be assessed in specially designed studies. Our study was designed to compare the short term effects of a centrally acting antihypertensive (rilmenidine) on blood pressure control, reversal of LVH, and lipid profile in contrast to other well established first choice antihypertensive drugs such as ACE inhibitors and CAAs.

In previous studies, rilmenidine was described as a metabolically inert antihypertensive drug since it had no negative effects on lipid profiles or glucose tolerance, or on other biochemical parameters ${ }^{19-21)}$ like other reference antihypertensive drugs except some diuretics. Even though our study indicates no significant changes in biochemical parameters during a 12 week period in all three treatment regimes, a statistically insignificant rise in HDL-C level $(+6 \%)$ was observed in the rilmenidine group.

In an epidemiologic analysis it was shown that an increment of $1 \mathrm{mg}$ per deciliter $(0.03 \mathrm{mmol} / \mathrm{L})$ or 2 to $3 \%$ in the HDL level has been associated with a reduction of 2 to $4 \%$ in the risk of cardiac events and is independent of the LDL level. ${ }^{22)}$ Whether our observation has a clinically important role and may be a special feature of imidazoline receptor agonists needs to be proven in large randomized long-term studies. De Luca, et al demonstrated that rilmenidine and amlodipine have comparable antihypertensive effects, but only rilmenidine was able to improve glucose metabolism in hypertensive patients with metabolic syndrome $\mathrm{X}^{23)}$

Both in sustained release form or the short-acting ordinary form, nifedipine, like perindopril, has been observed to result in a significant reduction of LVM in patients with essential hypertension. Also, an improvement in left ventricular diastolic function has been shown with these agents. ${ }^{7,24,25)}$ Our short-term results confirm these previous studies, however, we did not observe a significant improvement in the E/A ratio in patients receiving nifedipine in contrast to the perindopril and rilmenidine groups which showed significant improvements in diastolic function. Also, the ratio between the reduction in left ventricular mass and the fall in mean arterial pressure ( $\mathrm{LVM} / \mathrm{mmHg}$ ) was more pronounced in the perindopril and rilmenidine groups. This suggests that perindopril and rilmenidine may have additional effects on determinants of LVH other than lowering BP even in the short term. Both have a positive effect on sympathetic overactivity, which is a common underlying factor, and influenced all risk factors favourably, thereby protecting the patients from the complications of hypertension beyond that provided by a reduction in blood pressure. It was also reported that rilmenidine-induced regression of $\mathrm{LVH}$ was associated with a significant and persistent reduction in atrial natriuretic factor (ANF) levels indicating a favorable effect on the biosynthetic properties of the ventricular myocardium. ${ }^{3)}$ 
Data obtained from meta-analyses indicate that diuretics and ACE inhibitors are generally superior to other classes of antihypertensive drugs, especially calcium channel and beta receptor blockers. ${ }^{8)}$ Our study found better results with ACE inhibitor and selective imidazoline-1 receptor therapy compared to CAAs. Rilmenidine has comparable efficacy to that of superior first-line reference drugs such as ACE inhibitors and can be a reasonable alternative with few side effects and with enough potency to reverse the most important cardiac end-organ complication of hypertension.

In conclusion, despite its neutral effect on biochemical parameters and probable positive effect on lipid profiles, rilmenidine is as effective as the ACE-I perindopril and the CAA nifedipine in controlling hypertension. Rilmenidine decreases hypertension-induced left ventricular hypertrophy in the short term and has the ability to decrease left ventricular mass index as good as an ACE inhibitor and better than a CAA, not only due to the reduction in arterial BP, but also probably through its modulation of the sympathetic nervous system. The superior improvement in left ventricular diastolic function obtained by rilmenidine and perindopril in comparison to nifedipine-treated patients in a short time span may also be due to the restoration of pathological sympathetic overreactivity and improvement of cardiac structural changes.

We think that rilmenidine may be a reasonable alternative for patients with hypertension and $\mathrm{LVH}$, especially when drugs with proven vasculo-protective benefits such as ACE inhibitors cannot be used and seems to be a logical choice rather than a CAA for the treatment of complicated hypertension.

Further specifically designed studies should be conducted to evaluate the short and long term effects of antihypertensive medications, including rilmenidine, on lipid profiles and the influence of factors other than mere antihypertensive efficacy of the drugs in reversing LVH and other end-organ damage because the ability to reduce blood pressure is the main but not the only parameter that must be taken into account in decisions concerning antihypertensive therapy.

\section{REFERENCES}

1. Levy D, Garrison R J, Savage D, Kannel W, et al. Prognostic implications of echocardiographically determined left ventricular mass in the Framingham Heart Study. N Engl J Med 1990; 322: 1561-6.

2. Dahlöf B, Pennert K, Hansson L. Reversal of left ventricular hypertrophy in hypertensive patients. A metaanalysis of 109 treatment studies. Am J Hypertens 1992; 5: 95-110.

3. Safar M E. Rilmenidine : A novel antihypertensive agent. Am J Med 1989; 87: 24S-9S.

4. Trimarco B, Rosiello G, Sarno D, Lorino G, et al. Effects of one year treatment with rilmenidine on systemic hypertension induced left ventricular hypertrophy in hypertensive patients. Am J Cardiol 1994; 74: 36A-42A.

5. Sadowski Z, Szwed H, Kuch-Wocial A, Kubasik A, et al. Regression of left ventricular hypertrophy in hypertensive patients after one year of treatment with rilmenidine. J Hypertens Suppl 1998; 16: S55-62. 
6. Ostermann G, Brisgand B, Schmitt J, Fillastre JP. Efficacy and acceptability of rilmenidine for mild to moderate hypertension. Am J Cardiol 1998; 61: 76D-80D.

7. Franz IW, Ketelhut R, Behr U, Tonnesmann U. Time course of reduction in left ventricular mass during longterm antihypertensive therapy. J Hum Hypertens 1994; 8: 191-8.

8. Sehridan D. Regression of left ventricular hypertrophy: do antihypertensive classes differ? J Hypertens 2000; 18: S21-7.

9. Kirkendall WM, Burton MC, Epstein FM, Frens ED. Recommendations for human blood pressure determinations by sphyngomanometers. Circulation 1967; 36: 980-8

10. Reichek N, Devereux RB. Left ventricular hypertrophy: Relationship of anatomic, echocardiographic findings. Circulation 1981; 63: 1391-8.

11. Levy D, Wilson P W, Anderson K M, Castelli WP, et al. Stratifying the patient at risk from coronary disease: New insights from the Framingham Heart Study. Am Heart J 1990; 119: 712.

12. Tubau JF, Szlachcic J, Braun S, Massie BM. Impaired left ventricular function reserve in hypertensive patients with left ventricular hypertrophy. Hypertension 1989; 14: 1-8

13. Hartford M, Wilkstrand J, Wallenstin T, et al. Diastolic function of the heart in untreated primary hypertension. Hypertension 1994; 6: 329-38.

14. Strauer BE. Development of cardiac failure by coronary small vessel disease in hypertensive heart disease. J Hypertens 1991; 9: S11-21.

15. Messerli FH, Nunez ED, Nunez MM, et al. Hypertension and sudden death. Arch Intern Med 1989; 149: $1263-$ 7.

16. Dominiac P. Historic aspects in the identification of the I1-receptor and the pharmacology of the imidazolines and blood pressure control. Boston/lodrecht/London, Kluwer Academic Publishers 1994: 21-30.

17. Bousquet P. Imidazoline receptors: From basic concepts to recent developments. J Cardiovasc Pharmacol 1995; 26: S1-S6.

18. Beau B, Mahieux F, Paraire M, Laurin S, et al. Efficacy and safety of rilmenidine on arterial hypertension. Am J Cardiol 1998; 61: 95D-102D.

19. Scemama M, Fevrier B, Beucler I, Dairou F. Effects of rilmenidine on lipid balance in hyperlipidemic hypertensive patients. Ann Cardiol Angiol 1996; 45: 595-601.

20. Scemama M, Fevrier B, Beucler I, Dairou F. Lipid profile and antihypertensive efficacy in hyperlipidemic hypertensive patients: comparison of rilmenidine and captopril. J Cardiovasc Pharmacol 1995; 26 suppl 2: S349 .

21. De Luca N, Izzo R, Fontana D, Iovino G, Argenziano L, et al. Hemodynamic and metabolic effects of rilmenidine in hypertensive patients with metabolic syndrome X. J Hypertens 2000; 18: 1515-22.

22. Gordon DJ, Protsfield JL, Garrison RJ, et al. High density lipoptotein cholesterol and cardiovascular disease: four prospective American studies. Circulation 1989; 79: 8-15.

23. De Luca N, Izzo R, Fontana D, Ivino G, et al. Haemodynamic and metabolic effects in hypertensive patients with metabolic syndrome X. A double blind parallel study versus amlodipine. J Hypertens 2000; 18: 1515-22.

24. Yamakado T, Teramura S, Oonishi T, Maeda M, et al. Regression of left ventricular hypertrophy with long-term treatment of nifedipine in systemic hypertension. Clin Cardiol 1994; 17: 615-8.

25. Yalcin F, Aksoy FG, Muderrisoglu H, Sabah I, et al. Treatment of hypertension with perindopril reduces plasma atrial natriuretic peptide levels, left ventricular mass, and improves echocardiographic parameters of diastolic function. Clin Cardiol 2000; 23: 437-41. 